\title{
Cross-Border Governance in europäischer Regionalkooperation
}

\author{
Peter Ulrich und James W. Scott
}

\begin{abstract}
Der Beitrag gibt ausgehend von einem Interesse an Cross-Border Governance einen Überblick über Entwicklung, disziplinäre Verortung und definitorische Prämissen des Begriffs Governance hin zu einem der größten ,Modewörter' der Sozialwissenschaften. Es wird der Kontext und Entstehung von Cross-Border Governance beleuchtet, das Wechselverhältnis von Governance mit Grenze, Grenzregion und Institutionalisierung grenzüberschreitender Kooperation. Dazu werden vier Beispielstudien präsentiert und abschließend die Stärken und Schwächen des Begriffs besprochen.
\end{abstract}

\section{Schlagwörter}

Governance, Cross-Border Governance, grenzüberschreitende Kooperation, Regieren, Grenzmanagement

\section{Einleitung}

In der politischen Geografie wurde für kollektives Planen und Regieren der Terminus Governance eingeführt, der im weitesten Sinne Regieren jenseits des Nationalstaats (Zürn 2016) oder Regieren ohne Regierung (Rosenau 1992) durch dezentrale, nichthierarchische Netzwerke umschreibt. Der seit den 1990er-Jahren in den sozialwissenschaftlich geprägten Europa-, Grenz- und Regionalstudien sehr präsente Begriff kann dabei sowohl das Regieren im internationalen Mehrebenensystem (Multi-Level Governance), im Bereich der Außen- und Sicherheitspolitik (Border/Security Governance) oder der grenzüberschreitenden Zusammenarbeit (Cross-Border Governance) insbesondere der Europäischen Union (EU) beschreiben. Letzterem Anwendungsgebiet wollen wir uns mit diesem Beitrag zuwenden. Ausgehend von der Beobachtung, dass sich die Akteur*innennetzwerke des nationalstaatlichen Grenzmanagements zunehmend ausdifferenzieren und grenzüberschreitende (Regional-)Kooperationen dadurch zugenommen haben, lassen sich Fragen in Bezug auf die dahinterliegenden Politikund Planungsprozesse nicht (mehr) eindeutig beantworten. Wer regiert und steuert politische Grenzen? Wie wird über Grenzen regiert und wie werden politische Planungsprozesse über Grenzen hinweg gesteuert?

Der vorliegende Beitrag setzt sich zum Ziel, diese grenzüberschreitenden Prozesse unter dem Begriff der Cross-Border Governance zu beleuchten. Im Folgenden wird zunächst der Begriff Governance disziplinär verortet und entlang verschiedener Prämissen geschärft (Kapitel 2), bevor im Anschluss das Konzept Cross-Border Governance erklärt (Kapitel 3) und anhand von empirischen Beispielen im EU-Kontext erläutert wird (Kapitel 4). Abschließend wird es eine kritische Evaluation von Stärken, Grenzen und Weiterentwicklungsmöglichkeiten dieser Begrifflichkeit geben (Kapitel 5).

\section{Der Begriff Governance}

Es gibt zahlreiche verschiedene Definitionen des Begriffs Governance, die sich teilweise je nach Analysefokus und Disziplin stark unterscheiden. Differenzen tauchen etwa beim Basisverständ- 
nis (empirisch-analytisch oder normativ-ontologisch) oder bei der Theoriefähigkeit des Begriffs (Theorie, Metapher oder theoretisches Modell) auf.

Governance kann als Politik, als Prozess, als Struktur oder auch als Mechanismus für dezentrale Politik verstanden werden. Sie kann aber auch die Bedingung, Methode und Verfahrensweise von kollektiver Politikgestaltung oder governing practice bedeuten. Trotz uneinheitlicher Versuche, das Konzept Governance zu greifen, lassen sich doch einige wiederkehrende Prämissen festhalten, die folgendermaßen zusammengefasst werden können:

1. Governance (horizontal, bottom-up, nichthierarchisch, im Sinne von Regieren) ist abzugrenzen von Government (vertikal, top-down, hierarchisch, im Sinne von Regierung);

2. Governance umfasst eine Vielzahl an heterogenen Akteur*innen (staatlich, nichtstaatlich);

3. Governance ist ein hybrides Konzept, das politische Steuerung, Kontrolle, Regieren, Management oder Koordination bedeuten kann;

4. Governance ist häufig transnational ausgeprägt;

5. Governance umfasst eine wiederkehrende und unvollständige Form der Meinungs- und Entscheidungsfindung;

6. Governance kann funktional oder territorial verstanden werden;

7. Governance findet über verschiedene territoriale oder administrative Ebenen statt, die miteinander verflochten sind.

Diese sieben Prämissen von Governance sollen im Folgenden als Orientierung dienen, da keine einheitlich-einvernehmliche Definition existiert. Zudem geht dieser Beitrag von der am häufigsten postulierten Annahme des Begriffs Governance als einem theoretischen Modell aus, das zur Identifikation und Analyse von politischen Deliberations- und Entscheidungsprozessen (Politics) von mehreren Akteur*innen (Polity) über nationalstaatliche und administrative Grenzen hinweg, die ein gemeinsames Politikfeld, Programm, Projekt (Policy) oder Territorium entwickeln, nützlich ist.

\subsection{Kontext, Entstehung und disziplinäre Verortung}

Der Begriff Governance wurde in den 1980er-, spätestens in den 1990er-Jahren aus den Wirtschaftswissenschaften in weitere Disziplinen, etwa in die Politik- und Verwaltungswissenschaft, übertragen. Insbesondere in den European, Regional und Border Studies und den Internationalen Beziehungen hat er sich schnell etabliert. So wurde in der Institutionenökonomie, einem Querschnittsbereich zwischen Politik- und Wirtschaftswissenschaften, Governance als „an exercise in assessing the efficacy of alternative modes (means) of organization " (Williamson 1996, S. 11) beschrieben. Governance wird hier als eine neue Organisationsart klassifiziert, die auch ein Bündel von Bedingungen zur effizienteren Organisation von Bereichen, Sektoren und Aufgaben umfasst (vgl. Rhodes 1996, S. 658). Die Transformation vom wirtschaftlichoptimierenden Managementterminus zum normativ aufgeladenen Politikbegriff ist durch das Konzept Good Governance geschehen, das Empfehlungen für eine „gute“ Politikpraxis durch ein „Programm zur Verbesserung des Regierens in nationalen und internationalen politischen Systemen “ (Benz/Dose 2010, S. 20) vorschlägt. ${ }^{1}$ Auch im Bereich der Public Policy - also

1 Governance wird in diesem Zusammenhang einfach als Politik oder Anwendung von Politik verstanden. Die Grundannahmen, die später auch die Weltbank als offizielle guidelines definiert hat, sehen für good governance 
der Schnittstelle zwischen Politik- und Verwaltungswissenschaften - speziell in den European Studies ist der Begriff durch Multi-Level Governance (vgl. Hooghe/Marks 2003) oder EU Governance (vgl. Jachtenfuchs/Kohler-Koch 2010) stark präsent und dient seit den 1990erJahren als Modell zur Analyse und Beschreibung europäischer Politik- und Entscheidungsprozesse über Verwaltungs- und Landesgrenzen hinaus. Governance ist über Disziplinen hinweg ein Modewort geworden und ,gehörte um die Jahrtausendwende zu den Favoriten im Wettbewerb um den Titel des meistgenutzten Begriffes in den Sozialwissenschaften " (Blatter 2006, S. 50; vgl. Beck 2017, S. 351). Da es - wie bereits erwähnt - keine einheitliche Definition gibt, ist es wichtig, sich nochmal die zentralen Prämissen des Begriffs zu verdeutlichen.

\subsection{Prämissen des Governance-Begriffs}

Wie einleitend erwähnt, kann Governance entlang von einigen Prämissen spezifiziert werden, was im Folgenden etwas vertieft wird. Governance lässt sich als Antithese zum Begriff Government verstehen. Politische Steuerung von kollektivem Regieren im globalen Westen geschieht - so die dem Begriff innewohnende These - nicht hierarchisch top-down durch eine (nationalstaatliche) Regierung, sondern durch eine nichthierarchische, netzwerkartige Form des Regierens. Es wird auch häufig als Regieren außerhalb/neben/mit dem Nationalstaat aufgefasst, aber nicht durch den Nationalstaat allein, was auf die neofunktionalistischen Grundannahmen des Begriffs zurückzuführen ist (vgl. Knodt/Große Hüttmann 2012). Diese Grundannahmen fußen nicht auf der Überzeugung, dass der Nationalstaat abgeschafft wird, sondern in globalen Fragen immer unwichtiger und ohnmächtiger wird. Der Nationalstaat bleibt aber ein wesentlicher Teil in Governance-Strukturen und ist kooperativ tätig.

Durch Transnationalisierung, Denationalisierung und Globalisierung sind die Bearbeitung und Handhabung von globalen und europäischen wirtschaftlichen und politischen Zusammenhängen und transnationalen Flows hyperkomplex geworden. Der Nationalstaat - zumindest in westlichen Industrienationen - ist immer weniger in der Lage, diese komplexen Zusammenhänge allein zu lösen und delegiert Kompetenzen und Durchführungsakte an verschiedene, in Netzwerken organisierte Akteur*innen. Angesichts der Hyperkomplexität der internationalen Märkte, der Eigendynamik der globalen Wirtschaft und zunehmender internationaler Verflechtungen auf politischer Ebene setzt der Nationalstaat auf Kooperation mit supra-/subnationalen Organisationen - also der EU und den Regionen/Kommunen -, aber auch privaten wirtschaftlich tätigen Akteur*innen (vgl. Hungerland/Teupe in diesem Band). Trotz nationaler Grenzziehungsprozesse in Wirtschaft (Handelsblockaden und Strafzölle) und Politik (im Zuge von globalen Migrationsbewegungen oder der aktuellen Coronavirus-Pandemie) können viele soziale, wirtschaftliche und ökologische Sachverhalte nicht mehr im „nationalen Container“ (Sassen 2013) gelöst werden (vgl. Benz/Dose 2010, S. 15). Vielmehr wird der Fokus auf regelmäßige Formen von Gemeinschaftskooperationen von verschiedenen Akteur*innen ${ }^{2}$ und auf

einen effizienten öffentlichen Dienst, eine unabhängige Judikative und einen rechtlichen Rahmen vor (vgl. Rhodes 1996, S. 656).

2 Im Bereich der EU Multi-Level Governance kann nach Akteur*innen auf vertikaler Ebene (Institutionen auf supranationaler, nationaler und subnationaler Ebene) und horizontaler Ebene (Akteur*innen auf derselben vertikalen Ebene) unterschieden werden. So können auf der vertikalen Dimension Akteur*innen in GovernanceStrukturen etwa die EU-Kommission, das EU-Parlament, einzelne Parlamentarier, der Europäische Ausschuss der Regionen (supranationale Ebene), das Ministerium für Justiz oder das Bundesamt für Bau-, Stadt- und Raumforschung (nationale Ebene) und Regionen (in Deutschland die Bundesländer), Landkreise und Kommunen 
unterschiedliche transnationale Maßstäbe (Scales) oder administrative Ebenen (Layer) gelegt. Des Weiteren kann Governance funktional (kollektive Bearbeitung eines Problems, Projektes, Programmes, Politikbereiches oder einer spezifischen Aufgabe) oder territorial (Entwicklung eines Raums oder Region) ausgeprägt sein (vgl. Benz/Dose 2010). Auf regionaler Ebene können „netzwerkartige regionale Formen der Selbststeuerung unter Einbezug von Akteuren aus Politik, Verwaltung, Wirtschaft und/oder der Zivilgesellschaft“ (Fürst 2007, S. 356) entstehen. Im Bereich der Cross-Border Governance ist auch der Blick auf die A-/Symmetrie, also die diagonale Form der Governance, äußerst relevant und interessant.

\section{Das Konzept Cross-Border Governance}

Im Bereich der grenzüberschreitenden Kooperation ${ }^{3}$ spielt das Governance-Konzept eine herausragende Rolle. Es ist dann etwa die Rede von grenzübergreifender Governance oder CrossBorder Governance. Der Begriff hat sich in Europa ab Ende der 1990er-Jahre herausgebildet (vgl. Scott 1999; 2000a; 2002; Leresche/Saez 2002; Perkmann/Sum 2002; Gualini 2003; Kramsch/Hooper 2004; Perkmann 2007), um die Zunahme von Kooperationsprozessen über nationalstaatliche Grenzen hinweg zu markieren und zugleich einen analytischen Ansatz für die Beschreibung dieser Interaktionsprozesse über territoriale Grenzen hinweg zur Verfügung zu stellen. Im Folgenden wird der Hintergrund der Entstehung des Begriffs erläutert und Cross-Border Governance entlang definitorischer Merkmale eingeordnet.

\subsection{Kontext und Entstehung}

Im ersten Abschnitt wurde zu den zentralen Merkmalen des Governance-Begriffs erklärt, dass politische Steuerung nicht mehr auf nationaler Ebene, sondern zwischen den Ebenen auf globaler, supranationaler und subnationaler Ebene stattfindet. Im Bereich der grenzübergreifenden Kooperation an innereuropäischen Grenzen in der EU hat eine „Regionalisierung durch Europäisierung“ (vgl. Keating 2002, S. 215) Formen von grenzüberschreitender (CrossBorder) Governance geschaffen. Genauer gesagt wurde die grenzüberschreitende subnationale Ebene politisch, rechtlich und finanziell durch den Europarat, die EG (Europäische Gemeinschaft) und später die EU unterstützt. ${ }^{4}$ Gleichzeitig hat diese Entwicklung den Druck auf die Akteur*innen in Grenzlage erhöht, grenzüberschreitend aktiv zu werden, da es durch die Förderung des europäischen Subsidiaritäts- und Partnerschaftsprinzips ${ }^{5}$ von der EU entscheidende

(subnationale Ebene) sein. Auf horizontaler Ebene können diese Akteur*innen auf regionaler Ebene das Landesministerium für Europa oder Wirtschaft, die Industrie- und Handelskammern, die ortsansässigen Vereine, Interessensvereinigungen und Kammern, sowie zivilgesellschaftliche Initiativen sein, die sich z.B. in die gemeinsame Gestaltung von regionalen Entwicklungsplänen einbringen.

3 Das Konzept Cross-Border oder grenzüberschreitende Governance kam speziell im Rahmen der EU-Regionalpolitik, aber auch in anderen regionalen und globalen Kontexten auf.

4 Politisch wurde die grenzüberschreitende Zusammenarbeit in Europa durch die Einheitliche Europäische Akte (1987) und den Vertrag von Lissabon gefördert, u.a. mit der Stärkung der EU-Regionalpolitik und der Schaffung des Ausschusses der Regionen (1995). Rechtlich wurde die subnationale Ebene durch das Subsidiaritätsprinzip (1992) bestärkt und durch die Einführung des EU-Binnenmarktes und des Schengener Abkommens wirtschaftliche Zirkulation und Reisefreiheit über Grenzen gefördert. Finanziell hat vor allen Dingen die Gemeinschaftsinitiative INTERREG (1990) und später die Europäische Territoriale Zusammenarbeit (ETZ) viele Projekte, Programme und Politiken grenzüberschreitend auf subnationaler Ebene ermöglicht.

5 Das von der EU eingeführte Subsidiaritätsprinzip gibt untergeordneten staatlichen Organisationen hohe Selbstbestimmungsrechte. Das Partnerschaftsprinzip ist von der EU hauptsächlich im Rahmen der Regionalpolitik eingesetzt worden. 
Anstöße gab, die Kooperation mit den Nachbar*innen jenseits der Grenze zu vertiefen. Diese grenzregionale Förderung von Kooperationen hat zu einer Heterogenisierung von Netzwerken des Regierens in grenzüberschreitenden Regionen geführt. Speziell durch das INTERREG-Programm wurde in den 1990er-Jahren ein wahrer Boom grenzüberschreitender Zusammenarbeit ausgelöst. Angesichts der Entstehung von Kooperationsräumen über Grenzen hinweg stellt sich die Frage „[H]ow these emergent spaces are specifically to be governed? “ (Kramsch/Hooper 2004, S. 3). Politisch sind einige politische Absichtserklärungen zwischen angrenzenden Staaten geschlossen worden, grenzüberschreitend zu kooperieren. ${ }^{6}$ Institutionell entstanden seit den 1990er-Jahren zahlreiche sogenannte Euroregionen, Eurodistrikte oder Euregios ${ }^{7}$. Nach Markus Perkmann sind Euroregionen kleinskaligere Versionen von Grenzregionen, die auch als „micro-CBR "8 bezeichnet werden können und die mit territorialen Gebietskörperschaften über eine oder mehrere nationalstaatliche Grenzen hinweg einen Kooperationsverbund eingehen (vgl. Perkmann 2007, S. 861). Strategisch werden seit 1990 durch das EU-Programm INTERREG und später die Europäische Territoriale Zusammenarbeit (ETZ) von den zuständigen Verwaltungsbehörden siebenjährige Kooperationsprogramme herausgearbeitet, die mehrjährige Projekte finanzieren, um Grenzregionen wirtschaftlich und sozial zu stärken und die territoriale Kohäsion zu fördern. Diese Förderung von Grenzregionen und die dadurch entstandene erhöhte Notwendigkeit zur Steuerung und Planung grenzüberschreitender Prozesse führte zur Herausbildung von Governance-Strukturen über nationalstaatliche Grenzen hinweg.

In diesem Zusammenhang wurde in der wissenschaftlichen Literatur Cross-Border Governance als theoretisches Modell eingeführt, um die Organisation und Koordination von Regieren und politischer Steuerung in Grenzregionen im Bereich von öffentlichen Dienstleistungen (Bildungs-, Infrastruktur-, Gesundheits-, Energie- und Transportmaßnahmen) zu erfassen. Durch das Analysemodell wurde der Fokus konkret auf die Einbeziehung von verschiedenartigen Akteur*innengruppen gelegt, die zumeist das Ziel haben, bei durchlässigen (permeablen) nationalstaatlichen Grenzen grenzüberschreitend zu agieren.

\subsection{Definitorische Annäherung}

Grenzüberschreitende Governance oder auch Cross-Border Governance beschreibt und untersucht Strukturen und Prozesse der politischen Steuerung durch Netzwerke kollaborativen Regierens über nationalstaatliche Grenzen hinweg. Das Konzept fokussiert dabei meistens die subnationale, also regionale und lokale Ebene im Hinblick auf Entwicklungsaspekte eines konkreten Raums oder Sachverhalts. Im europäischen Integrationsprozess geht es dabei primär um die politische Steuerung und das kollektive Regieren durch verschiedene staatliche und nichtstaatliche Akteur*innen (öffentliche und private Organisationen) sowie grenzüberschrei-

6 Ein Beispiel dafür ist der deutsch-polnische Nachbarschaftsvertrag (kurz für „Vertrag zwischen der Bundesrepublik Deutschland und der Republik Polen über gute Nachbarschaft und freundschaftliche Zusammenarbeit") vom 17.6.1991, der Initialzünder der deutsch-polnischen Zusammenarbeit war und einige Politikbereiche als gemeinsame Kooperationsfelder benennt. Siehe dazu auch Ulrich (2017, S. 375).

7 Die erste Euregio ist zwar 1958 an der deutsch-niederländischen Grenze entstanden, allerdings ging der Boom der Gründungen weiterer Euroregionen erst nach 1990 los. Das liegt zum einen an der neuen europäischen Gemeinschaftsinitiative INTERREG, die grenzüberschreitende Kooperationsprogramme und -projekte großzügig finanziell unterstützt hat, zum anderen hängt es aber auch mit dem Fall des Eisernen Vorhangs zusammen und der Heranführung des europäischen Ostens an die westeuropäischen Länder. Nach Sara Svensson (2013) existieren in Europa heute etwa 150 Euroregionen, die meisten wurden nach 1990 gegründet.

8 Nach Perkmann (2007, S. 861). CBR ist die Abkürzung von Cross-Border Region (Grenzregion). 
tend-supraregionale Akteur"innen (grenzüberschreitende Institutionen) über verschiedene Verwaltungsebenen und konkret über mindestens eine nationalstaatliche Grenze hinweg. Auch wenn der Begriff um die Jahrtausendwende in zahlreichen Beiträgen prominent diskutiert wurde, gibt es - wie bereits erwähnt - kaum konkrete Definitionen dazu. Im Folgenden werden die wenigen Definitionen, die es im Bereich der europäischen Grenzraumstudien gibt, kurz skizziert.

Cross-Border Governance kann in Bezug auf die grenzüberschreitende EU-Regionalpolitik und im Rahmen der EU-Integrationsprozesse entlang der politisch-ökonomischen, institutionellen und symbolisch-kognitiven Dimension unterschieden werden (vgl. Gualini 2003, S. 44ff.). Der Begriff wird nach Enrico Gualini eher in einem institutionalistischen Verständnis definiert:

„Cross-border Governance is an institutional construct resulting from complex processes of co-evolution. In their current phase of institutionalisation, cross-border Governance settings face a struggle that highlights the dialectics between path-dependency and pathshaping, between institution building and institutional design“ (ebd., S. 43).

Das Entstehen von grenzüberschreitenden Governance-Strukturen ist daher stets mit Bedingungen des Gelingens und Pfadabhängigkeiten verbunden, die in den drei oben genannten Ebenen verortet sind.

Des Weiteren wird Cross-Border Governance häufig als Resultat der Compliance mit EURechtsprechung durch öffentliche Akteur*innen und territoriale Gebietskörperschaften verstanden. Diese Governance-Strukturen entstehen durch Prozesse der sogenannten ,Europäisierung' von öffentlichen Verwaltungen und regionalen Behörden, wenn nämlich Normen, Strategien und Rechtsprechungen von der EU auf diese Organisationen übertragen werden. Noralv Veggeland beschreibt dieses Zusammenwachsen folgendermaßen:

„Externally, the dynamic of spatial multilevel Governance formations is structured by both upwards and downwards pooling of sovereignty into vertical EU-state-region partnerships. Internally, the domestic regional tier develops its own local partnerships in a horizontal order. Furthermore, the growing number of cross-border jurisdictions and transboundary region as partnership constructions, i.e. the EU-regions and the Euro-regions, are organized in a horizontal order. [...] As partners, the cooperating regions, the states, the private actors, and the EU, as indicated above, have all subordinated themselves under the legal rules of negotiated treaties, agreements and contracts. Thus, the building of EU-regions and the Euro-regions are intimately linked to EU Governance, to the arising territorial multilevel Governance and partnership structures in Europe“ (Veggeland 2004, S. 161f.).

Hier wird deutlich, dass das Konzept Cross-Border Governance teilweise von anderen Formen der Governance in der EU, insbesondere der Multi-Level Governance, schwer zu trennen ist. Zudem wird das Konzept, wie bereits aufgezeigt, als Konsequenz der EU-Rechtsprechung und der Einhaltung von Rechten und Normen der EU gesehen.

Jarosław Jańczak verwendet das Konzept der Cross-Border Governance als Steuerungsbegriff in Folge der immer stärker werdenden Entgrenzung und Verflechtung im Grenzraum durch Europäisierungsprozesse: „De-bordering (but also re-bordering) and increasing (or decreasing) mutual interdependencies create a necessity for cross-border Governance of the traditional public sphere where all the actors can be involved" (Jańczak 2011, S. 39f.). Wie auch bei Veggeland (2004) wird Cross-Border Governance hier als Antwort auf „transformative Prozesse“ 
verstanden. Tarmo Pikner erweitert diese Bestimmung, wenn er Cross-Border Governance als eine „social infrastructure across the state borders which create channels for the transfer or flow of material and non-material resources“ (Pikner 2008, S. 2014) beschreibt. Ein solches Verständnis von Governance betont die Bedingungen der Ermöglichung von Zirkulation von Objekten, Subjekten, Wissen, Kapital etc. Des Weiteren spricht James Scott von transnationaler Governance, einem Konzept, das dezentralisierte Formen der Interaktion über Grenzen umfasst (vgl. Scott 1999, S. 606) und auf angemessene Art und Weise auf Probleme reagiert, die zuvor nicht effizient und effektiv genug gelöst wurden:

"Clearly then, one of the central issues determining the significance of transnational regionalism is that of Governance, of addressing in a politically meaningful way local and regional concerns that transcend traditional national and/or international problem-solving capacities“ (Scott 2002, S. 136).

In dieser Definition wird nochmal die eingangs erwähnte Prämisse des Konzepts deutlich, dass Governance ein Problemlösungsmodus sein kann, wenn der Nationalstaat alleine nicht in der Lage ist, diese Probleme zu lösen. Nachdem die definitorische Annäherung an den Begriff vollzogen worden ist, wird im Folgenden der Grenzbezug hervorgehoben.

\subsection{Grenzbezug}

Anders als Global oder Multi-Level Governance, die auf unterschiedliche Skalen und administrative Ebenen verweisen, legt der Cross-Border Governance-Ansatz den Fokus explizit auf die räumlich-territoriale Dimension. Zudem besteht ein klarer Grenzbezug. Wie der Begriff grenzüberschreitende/Cross-Border Governance verdeutlicht, geht es hier um eine Form der Politiksteuerung und um Interaktionen über nationalstaatliche Grenzen hinweg. Der Fokus liegt also ganz klar auf politisch-territorialen, zumeist nationalstaatlichen Grenzen. Im Fall der EU muss zwischen inneren und äußeren territorial-politischen Grenzen unterschieden werden.

Governance an oder über innere EU-Grenzen hinweg wird häufig im Rahmen der EU-Regionalpolitik durchgeführt. Demnach handelt es sich um regionale Formen der grenzüberschreitenden Zusammenarbeit, etwa in den Bereichen der Daseinsvorsorge, wie Gesundheit, ÖPNV, Bildung, aber auch Umweltschutz, Regional- und Stadtplanung und wirtschaftliche Kooperation (vgl. Eigmüller in diesem Band). Governance an und über äußere EU-Grenzen hinweg umfasst eher die hoheitlichen Bereiche der Sicherheits- und Außenpolitik und die politische Steuerung an den Außengrenzen. Auch hier kooperieren verschiedene (z.B. staatliche und private) Akteur*innen unter Verwendung unterschiedlicher Technologien und transnationaler Informationsdatenbanken sowie EU-Einrichtungen wie die umstrittene Grenzschutzagentur Frontex miteinander (vgl. Pötzsch in diesem Band). Während also Cross-Border Governance über EU-Binnengrenzen hinweg im Dienste des Debordering steht, insofern Grenzüberschreitung erwirkt und so dem Wunsch nach permeablen Grenzen gefolgt wird, geht es im Falle der EU-Außengrenzen eher um Rebordering, insofern die Grenzsicherheit mit dem normativen Ziel der Schaffung von durablen Grenzen im Fokus steht. Für die Governance entlang der EU-Außengrenze wird im Rahmen der Diskussion um die Festung Europa auch häufig das Konzept Grenzregime verwendet (vgl. Hess/Kasparek 2010; siehe auch Hess/Schmidt-Sembdner in diesem Band).

Grenzen werden in beiden Fällen nicht als einfache Trennlinien, sondern territoriale Gebilde verstanden, welche die zwischennationalen Grenzen (innere EU-Grenzen) oder geopolitisch- 
systemischen Grenzen (äußere EU-Grenzen) überlagern und in dieser räumlichen Ausdehnung ein Netzwerkgeflecht an verschiedenen Akteur*innen und administrativen Ebenen verbinden. Im Folgenden wird der Fokus auf die institutionelle Dimension von Cross-Border Governance gelegt.

\subsection{Institutionalisierung von Grenzregionen}

Nach Gualinis Klassifizierung umfasst Governance auch eine Institutionalisierung grenzüberschreitender Zusammenarbeit (vgl. Gualini 2003, S. 44). Im Kontext von politischer Steuerung kann Governance daher aus einem formellen oder informellen Regelwerk zur Strukturierung und Konsolidierung grenzüberschreitender Politikpraxis bestehen. Jean-Philippe Leresche und Guy Saez verweisen darauf im Hinblick auf die grenzüberschreitende Zusammenarbeit in der EU: „Cross-border cooperation [...] is one of those objects which, by construction, requires simultaneously to consider productive systems, affiliations and political organization " (Leresche/Saez 2002, S. 77). Eine solche Institutionalisierung grenzüberschreitender Governance zielt auch auf eine Form supraregionaler Institutionenbildung, also einer überregionalen Einheit, die politisch nicht alleinig einer der beiden nationalen Gebietskörperschaften zuzurechnen ist, sondern über eigene grenzüberschreitende Kompetenzen verfügt. Im Hinblick auf Governance und Institutionen ist nach folgenden Bezügen zu differenzieren (vgl. Abbildung 1):

\section{Governance und Institutionen}

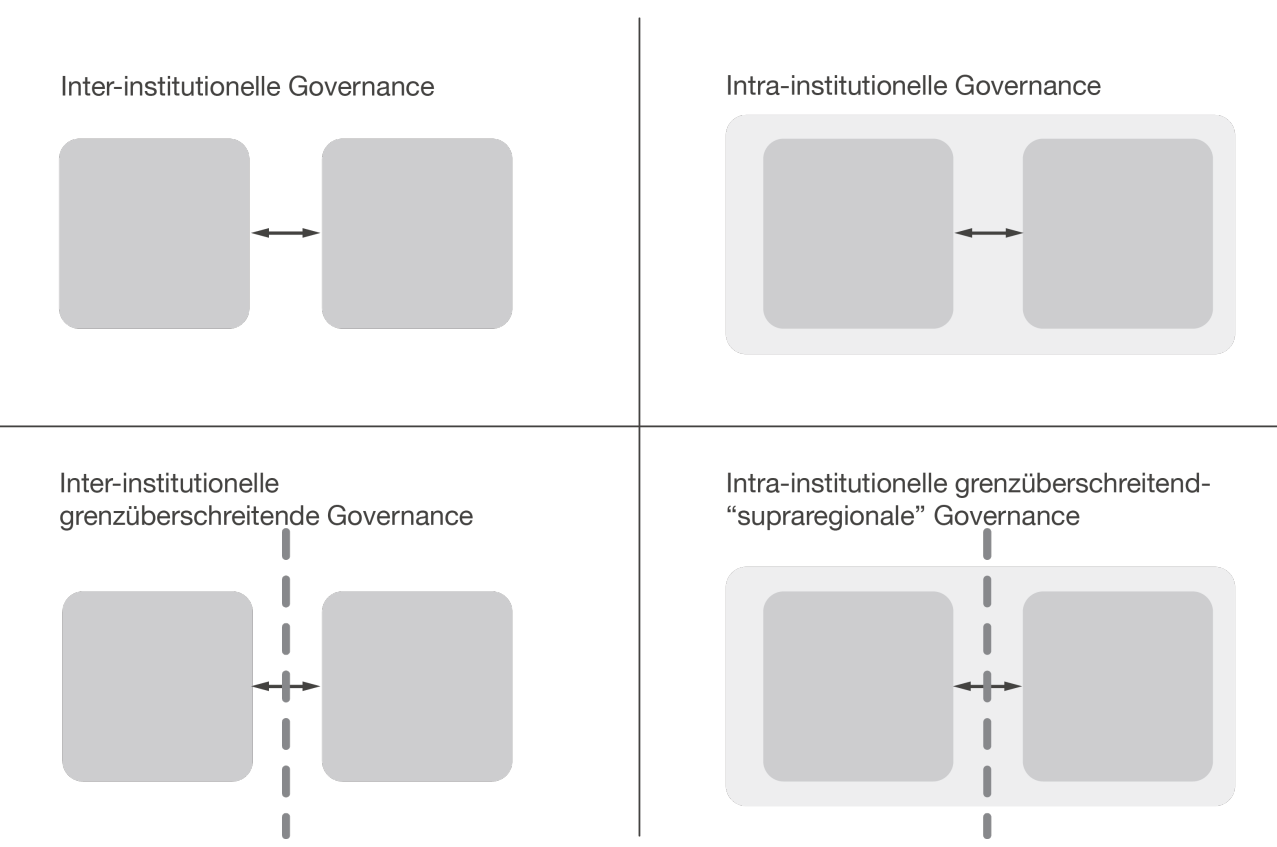

Abbildung 1: Governance und Institutionen (eigene Darstellung: Peter Ulrich)

Erstens beschreibt inter-institutionelle Governance innerstaatliche und zwischeninstitutionelle Formen der politischen Steuerung und Koordination. Funktionale oder territoriale Governance umfasst dabei mindestens zwei verschiedenartige Institutionen. Ein Beispiel dafür wäre im regionalen Kontext die politische Steuerung von Strukturwandel z.B. in der Lausitz, der von 
staatlichen, wirtschaftlichen, wissenschaftlichen und zivilgesellschaftlichen Akteur*innen koordiniert wird.

Zweitens beinhaltet intra-institutionelle Governance die innerstaatliche politische Steuerung zwischen mindestens zwei Akteur*innentypen innerhalb einer gemeinsamen Institution. So könnten zwei verschiedene Interessen innerhalb einer Organisation kollidieren und eine politische Steuerung vonnöten machen. Ein Beispiel sind regionale Innovations- und TechnologieCluster, die für die effiziente und effektive Steuerung von Entwicklungsprozessen innerhalb des Clusters verschiedene Einheiten umfassen, z.B. für Forschung sowie die Abteilung Transfers/Entwicklung. Hier bedarf es einer intra-institutionellen Steuerung - einer Governance von verschiedenen Organisationseinheiten.

Drittens verweist inter-institutionelle grenzüberschreitende Governance auf politische Steuerung von mindestens zwei Einrichtungen, die sich in unterschiedlichen Ländern, meist innerhalb einer Grenzregion, befinden. So können bei der Ausarbeitung eines mehrjährigen INTERREG-Programms z.B. zwischen Brandenburg und Polen regionale, lokale und nationale Entscheidungsträger auf beiden Seiten der Grenze in Interaktion treten.

Viertens bezieht sich intra-institutionelle grenzüberschreitend-supraregionale Governance auf die höchste Form der Institutionalisierung grenzüberschreitender Governance, da überregionale, d.h. supraregionale Strukturen geschaffen werden. Genauer gesagt beschreibt das Konzept die interne politische Steuerung von grenzüberschreitenden Institutionen. Eine weiche Form ist der eher informelle Rahmen der Euroregion, der ein Dach, basierend auf einer institutionellen Kooperationsvereinbarung zweier Trägervereine auf beiden Seiten der Grenze, darstellt. Eine formellere Form der Institutionalisierung supraregionaler Governance ist die Schaffung von rechtlich-verbindlichen Rahmen, etwa durch die Anwendung des EU-Instruments des Europäischen Verbunds für territoriale Zusammenarbeit (EVTZ) in Grenzregionen. Die intrainstitutionelle Governance in diesen supraregionalen Strukturen beinhaltet die Nutzung und das Zusammenspiel von gemeinsamen Organen, Bilingualismus in internen Kommunikationsprozessen sowie die gleichmäßige und bilinguale Besetzung von Spitzenpositionen und Personalstellen, um die Berücksichtigung der mindestens zwei nationalen Seiten im Grenzraum zu garantieren.

Die letztgenannte Form von Cross-Border Governance beschreibt Markus Perkmann mit Blick auf Euroregionen mit folgenden Eigenschaften: Grenzüberschreitende Governance umfasst mehrheitlich Akteur*innen aus dem öffentlichen Bereich (auch wenn andere Sektoren inkludiert werden), sie sind in informellen oder quasi-juridical Institutionen organisiert und dienen dem praktischem Problemlösen (vgl. Perkmann 2007, S. 863). Die grenzüberschreitenden Institutionen wie Euroregionen werden zumeist durch binationale Mitgliederräte, Präsidenten, $\mathrm{Ar}$ beitsgruppen und Sekretariate organisiert (vgl. ebd., S. 863). In den Räten und Arbeitsgruppen sind verschiedene Akteur*innengruppen vertreten - primär öffentlich-territoriale Akteur*innen -, aber auch private und zivilgesellschaftliche Netzwerke.

\subsection{Akteur*innenbezug und politikfeldspezifischer Anwendungsbezug}

Cross-Border Governance ist ein akteur*innenzentrierter Ansatz. Wie im Falle der Multi-Level Governance können Akteur"innen auf verschiedenen administrativen Ebenen (supranationale, nationale, subnationale Ebene) angesiedelt sein (vertikale Dimension). Das Besondere bei dem Ansatz der Cross-Border Governance ist aber der explizite Grenzbezug, der es erfordert, nicht 
nur auf Akteur*innen-Interaktionsmuster auf einer Seite der Grenze zu schauen (horizontale Dimension), sondern auch das Wechselverhältnis mit Akteur*innen auf der anderen Seite der Grenze im Blick zu haben. Hier geraten mitunter Asymmetrien in den Fokus, insofern über Grenzen kooperierende Akteur*innen sich auf unterschiedlichen administrativen Ebenen befinden (diagonale Dimension). So verfügen diese Akteur*innen über ähnliche oder ganz andere Formen von Kompetenzen (vgl. Maier 2009, S. 459).

Daher können zwei Besonderheiten in Bezug auf die Akteur*innenstrukturen der Cross-Border Governance genannt werden. Erstens besteht eine Art ,Spiegel' der Akteur"innenstruktur auf beiden Seiten der nationalstaatlichen Grenze. So können auf der regionalen oder lokalen Ebene verschiedene oder ähnliche Arten von Akteur*innengruppen grenzüberschreitend in Interaktion treten. Zweitens kann ein diagonales Verhältnis bei ungleich verteilten Kompetenzen und Ressourcen bei den territorialen Gebietskörperschaften zwischen Land A und Land B bestehen. Je nach Staatsorganisation (dezentral/föderalistisch vs. zentralistisch) liegen die zuständigen Behörden in Bezug auf spezifische Politikbereiche auf verschiedenen hierarchischen Ebenen. Die Governance über Grenzen hinweg wird dadurch asymmetrisch und diagonal, was Governance-Prozesse erschweren kann (vgl. ebd., S. 459).

Akteur*innen der Cross-Border Governance im Kontext der grenzüberschreitenden Kooperation in der EU gehören in erster Linie dem öffentlichen Sektor an und sind meist im Bereich der Daseinsvorsorge (Bildung, Gesundheit, Transport, Energie etc.) tätig. Weitere Akteur*innen sind etwa kulturelle, soziale und zivilgesellschaftliche Organisationen oder Interessensvertreter*innen aus dem privaten und wirtschaftlichen Bereich, die aber nach EU-Beihilfenrecht nicht problemlos EU-Regionalfondsmittel einwerben können. Normalerweise

„sind es daher kommunale und staatliche Verwaltungsakteure oder sonstige institutionelle Akteure wie Kammern (IHK, HWK) und Verbände des Dritten Sektors, die in der grenzüberschreitenden Zusammenarbeit tätig sind und die Projekte und Programme aus öffentlichen Budgets kofinanzieren“ (Beck 2017, S. 354).

Auch Perkmann geht von einem heterogenen Netzwerk an Akteur*innen aus, die aber mehrheitlich aus dem öffentlichen Bereich kommen:

„In most cases, the participating bodies are local authorities, although sometimes regional or district authorities are involved. Occasionally, other organizations, such as regional development agencies, interest associations and chambers of commerce also participate in the Governance of the CBR" (Perkmann 2007, S. 863).

Jańczak systematisiert die Akteur*innenstrukturen des Cross-Border Governance entlang einer vertikalen und horizontalen Form. Die horizontale Ebene bezieht sich hier auf Interaktionsprozesse verschiedener Akteur*innen auf beiden Seiten der Grenze. Wie auch in Abbildung 2 gezeigt, unterscheidet er zwischen Top-down- und Bottom-up-Formen der grenzüberschreitenden Governance:

„Consequently, cross-border Governance has two dimensions. Vertically it contains three levels: administration, NGOs and individuals that interact in the process, of formulating policies addressed to the partner town. Horizontally, on the other hand, the corresponding actors interact at each level. Cross-border Governance in a border twin town may be top-down (by administration) or bottom-up (by individuals) inspired, but it always requires collaboration of at least two actors from both sides of the border, representing the same level of Governance“ (Jańczak 2011, S. 41f.). 


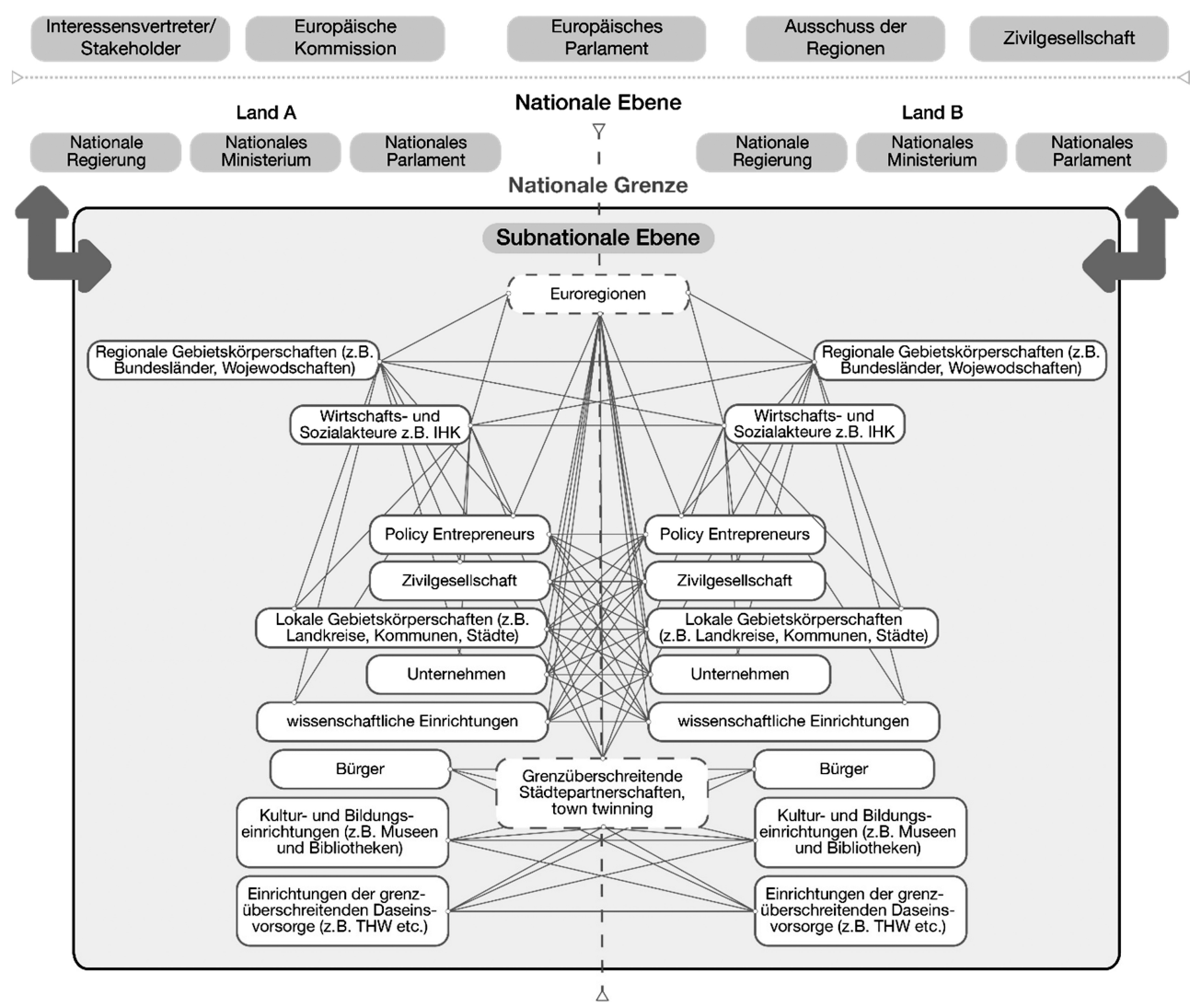

Abbildung 2: Das Modell Cross-Border Governance (eigene Darstellung: Peter Ulrich)

Die Ermöglichung innovativer, sozialer und von Bottom-up-Formen der grenzüberschreitenden Politikgestaltung bietet auch Raum für Beteiligung von neuartigen Akteur*innen, die sich über die Zeit auf diese Art von grenzüberschreitender Regionalpolitik spezialisieren. Perkmann bezeichnet diese Akteur*innen als policy entrepreneurs (vgl. Perkmann 2007, S. 862).

Ob Cross-Border Governance aber tatsächlich bottom-up geschieht, wie es von Perkmann oder Jańczak formuliert wird, ist nicht ausgemacht. Mit dem politischen, von Eliten geschaffenen Rahmen, bestehend aus regionalen, nationalen und europäischen Akteur*innen, ist ein Handlungsraum geschaffen, der sich nach europäischem Vorbild institutionalisiert und zusammenwächst: „Faktisch wird die Institutionalisierung grenzüberschreitender Zusammenarbeit jedoch nicht in die Hände derer gelegt, die vor Ort leben, also spontanen Prozessen der Selbstorganisation überlassen, sondern durch regionale Eliten oder europäische Vertreter stufenweise verankert“" (Banse 2013, S. 84). Daher kann Cross-Border Governance als eine Form der politischen Steuerung der Eliten verstanden werden, welche aber auch zahlreiche andere Akteur*innen zulässt.

Gegenstandsbereich politischer Steuerung durch eine Vielzahl von Akteur"innen sind im Bereich der grenzüberschreitenden subnationalen Kooperation das gemeinsame Planen, Entwerfen und Umsetzen von EU-Regionalpolitik in Grenzregionen (vgl. Klatt in diesem Band). EU-Regionalpolitik umfasst den Bereich der low politics, die in erster Linie die Grundsiche- 
rung der Bevölkerung und deren Daseinsvorsorge betrifft. Politikbereiche der EU-Regionalpolitik umfassen alle Bereiche der wirtschaftlichen, sozialen und territorialen Kohäsion, in der „Raumordnung, im Umweltschutz, beim öffentlichen Personennahverkehr (ÖPNV), in der Bildung, Forschung und Innovation" (Beck 2017, S. 354). Zu trennen ist der Bereich etwa von der Cross-Border Security Governance oder Foreign \& Security Governance, die sich als high politics um die Sicherung des Nationalstaates kümmern und somit vom Begriff der Cross-Border Governance klar zu differenzieren sind. Nach der Darlegung von zentralen Eigenschaften von grenzüberschreitender Governance werden im Folgenden einige Beispielstudien präsentiert.

\section{Anwendungsbeispiele und empirische Studien}

Im Folgenden wird die Anwendung des theoretischen Modells Cross-Border Governance anhand von vier empirischen Studien zum deutsch-polnischen Grenzraum illustriert. Obwohl es unzählige Governance-Fallstudien an Grenzen weltweit gibt, konzentrieren sich die meisten Studien auf EU-Grenzregionen, da im Kontext der Europäisierung und Regionalisierung ein besonderer Fokus und Bedarf an politischer Steuerung durch das postulierte Partnerschaftsprinzip in europäischen Grenzregionen vorliegt. Die deutsch-polnische Grenze wurde aufgrund ihrer starken politischen, historischen und sprachlichen Divergenzen und aufgrund der langjährigen Forschungstätigkeiten der Autoren in dieser Region als Analyseraum ausgesucht. Die Fallstudien in der Untersuchungsregion lassen sich nach verschiedenen Skalenniveaus differenzieren: Das erste Fallbeispiel umfasst die Doppelstadt Frankfurt (Oder)-Słubice (vgl. Jańczak 2011), das zweite Fallbeispiel den (euro)regionalen Verbund der Euroregion Pro Europa Viadrina (vgl. Perkmann 2007) und das dritte Fallbeispiel die transnationale Makroregion des Ostseeraums (vgl. Scott 2002). Das vierte Fallbeispiel zeigt mit der TransOderana EVTZ (in Gründung) eine besondere Form der Institutionalisierung von grenzüberschreitender deutschpolnischer Regionalkooperation (vgl. Ulrich 2017).

\subsection{Fallbeispiel 1: Doppelstadt und Kooperationszentrum Frankfurt (Oder)-Słubice}

In der Studie von Jarosław Jańczak (2011) zur Doppelstadt an der Oder wird gezeigt, wie mit dem Geschichtsverlauf seit 1945 Grenzverschiebungen, Bevölkerungswanderungen und -umsiedlungen zahlreiche geschichtliche und soziokulturelle Spuren in der Grenzregion hinterlassen haben, die auch in Zeiten der deutsch-polnischen Versöhnung die heutige Planung und grenzüberschreitende Kooperation nachhaltig beeinflussen. Zudem skizziert er verschiedene Formen von Asymmetrien - z.B. sprachliche, kulturelle, wirtschaftliche und soziale -, die die grenzüberschreitenden Politik- und Planungsprozesse vor Herausforderungen stellen (vgl. ebd., S. 43f.; siehe zu sprachlichen Grenzziehungen auch Nekula in diesem Band). So sind die soziale Integration und das Wissen über ,die Anderen' auf beiden Seiten der Grenze wenig ausgeprägt. Zudem wurden grenzüberschreitende Vorhaben wie etwa eine Tramlinie über die Grenze von der Bevölkerung kritisch gesehen und per Bürgerinitiative abgelehnt (vgl. ebd., S. 44). Ausgehend von diesen Rahmenbedingungen der deutsch-polnischen Grenzgeschichte für die Kooperation in der grenzüberschreitenden Doppelstadt, geht Jańczak im Folgenden auf verschiedene Akteur*innengruppen ein. Er identifiziert eine ausgeprägte Teilhabe und breites Engagement von Nichtregierungsorganisationen (NGOs) und zivilgesellschaftlichen Akteur*in- 
nen beiderseits der Grenze trotz Unterschieden in den Entwicklungen der Zivilgesellschaft auf beiden Seiten der Grenze (vgl. ebd., S. 44). Laut Jańczak betonen zivilgesellschaftliche Akteur*innen wie etwa die Stubfurt-Initiative ${ }^{9}$ die kulturellen, historischen und sozialen Gemeinsamkeiten, die die Grenze als ein verbindendes, weniger als ein trennendes Element in Szene setzt, und zielen durch Kollaboration und Interaktion auf die Optimierung von lokalen Problemlösungskapazitäten ab (vgl. ebd., S. 44). Die weitreichendste Form der Zusammenarbeit findet aber zwischen den Stadtverwaltungen statt. Diese „top-down organized Governance“ (vgl. ebd., S. 44), die durch die nationalen Hauptstädte und EU-Förderungen lange unterstützt worden ist, hat auf der supraregional-grenzüberschreitenden intra-institutionellen GovernanceEbene zahlreiche politische Steuerungsformen und -formate errichtet, wie gemeinsame Kommissionen, Sitzungen etc. Gleichzeitig wurden viele gemeinsame Symbole geschaffen, wie ein gemeinsames Stadtmarketing und -logo. Außerdem wurden zahlreiche grenzüberschreitende Projekte durchgeführt, etwa in den Bereichen Bildung, Sport, Raumplanung etc. (vgl. ebd., S. 44). Im Jahr 2010 wurden zudem ein Kooperationszentrum der Doppelstadt (2010) mit monatlichen gemeinsamen Sitzungen der Bürgermeister eingerichtet, zwei grenzüberschreitende Handlungspläne verabschiedet (2010-2020 und 2020-2030) und eine grenzüberschreitende Buslinie und ein Fernwärmesystem installiert (vgl. Ulrich/Krzymuski 2018, S. 167ff.).

Die Studie von Jańczak zeigt, dass grenzüberschreitende Governance zum einen ein Analysemodell ist, um regionalpolitische Prozesse zu beschreiben, zum anderen kann es auch als normatives Steuerungsinstrument verstanden werden, um verschiedene Akteur*innen über Grenzen hinweg für kooperative Zwecke zu verbinden - mit dem Ziel, historische Konfliktpotenziale abzubauen und neue Formen des Zusammenlebens zu gestalten. Die grenzüberschreitende Governance wurde 2010 durch eine Institutionalisierung der Zusammenarbeit zu einer supraregional-grenzüberschreitenden intra-institutionellen Governance-Form.

\subsection{Fallbeispiel 2: Euroregion Pro Europa Viadrina}

Die Euroregion, die den Ostteil des Landes Brandenburg und Teile der Wojewodschaft Lubuskie umfasst, wurde im Jahr 1993 mit damals noch starken Grenzdifferenzen (vgl. Perkmann 2007, S. 869) gegründet. Auf der deutschen Seite führten zwei Hauptmotive zur Gründung der Euroregion: Zum einen gab es zivilgesellschaftliche Ambitionen mit dem Ziel, einen Beitrag zur deutsch-polnischen Versöhnung bezüglich der Kriegsverbrechen im Zweiten Weltkrieg zu leisten, da sich mit der europäischen Integration nach dem Fall des Eisernen Vorhangs eine Möglichkeit dafür bot. Zum anderen war auch die Aussicht auf eine Regionalförderung durch den neu gegründeten Fördertopf INTERREG ein Motiv zur Gründung (vgl. ebd., S. 869f.). Das Land Brandenburg hat sich für die Schaffung einer Euroregion stark eingesetzt, die INTERREG-Mittel für Brandenburg und Polen teilweise für die Kooperation im genannten Territorium verwalten kann. In der Analyse der deutsch-polnischen Euroregion von Perkmann wird zum einen die inter-institutionelle grenzüberschreitende Governance anhand der beteiligten Akteur*innen aus Deutschland und Polen wie öffentlicher Behörden, funktionaler und repräsentativer Körperschaften (z.B. IHK) und des World Trade Center in Frankfurt (Oder)

9 Die Stubfurt-Initiative ist ein Kulturprojekt, das von einem Künstler in der Doppelstadt Frankfurt-Słubice geschaffen worden ist und auch aus einem Förderverein besteht. Zahlreiche Projekte der Stubfurt-Initiative zielen darauf ab, die in zwei Länder geteilte Stadt an der Oder als eine Einheit mit eigener Geschichte, Politik und kultureller Prägung zu sehen. 
beschrieben (vgl. ebd., S. 869). Zum anderen wird die intra-institutionelle grenzüberschreitend-supraregionale Governance, also die politische Steuerung innerhalb der Euroregion Pro Europa Viadrina veranschaulicht, die aus einem deutsch-polnischen bzw. euroregionalen Rat, der Präsidentschaft, einem Sekretariat und mehreren sektorspezifischen Arbeitsgruppen besteht (vgl. ebd., S. 869). Damit zeigt diese Studie zum einen, dass Governance ein akteur*innenzentrierter Begriff ist, der die Untersuchung von Interessen und Interaktionen einer Vielzahl von staatlichen und nichtstaatlichen Organisationen anleiten kann. Zum anderen offenbart sie auch, dass Governance immer in unterschiedlichen Kontexten und institutionellen Rahmen zu denken ist: Governance-Formen erscheinen in intra-institutionellen supraregional-grenzüberschreitenden Einheiten, wie in einer Euroregion durch gemeinsame Organe, können aber auch zwischen Organisationen im Grenzraum in Form inner-institutioneller grenzüberschreitender Governance-Prozesse vorkommen.

\subsection{Fallbeispiel 3: Makroregion Ostseeraum}

Im Unterschied zu den beiden Studien, die Governance von kleinteiligen Regionen oder Städte an der deutsch-polnischen Grenze untersuchen, analysiert folgende Studie Governance-prozesse und -strukturen in Makroregionen, also großflächige transnationale Räume, die neben deutschen und polnischen Regionen auch weitere Länder umfassen. In der Studie von James Scott (2002) geht es konkret um die Makroregion des Ostseeraums (Baltic Sea Region), die aus „subregionalen Kernen der Kooperation aus mehrheitlich nordeuropäischen Staaten“ (vgl. ebd. S. 137) besteht und sich über die durch den europäischen Integrationsprozess zusammenwachsenden Regionen aus den baltischen Staaten, Ostdeutschland, Polen und Russland definiert (vgl. ebd., S. 137). Die Studie beleuchtet primär Formen und Foren der politischen Steuerung und Koordination von verschiedenen Skalen und Formen der Kooperation in multilateralen transnationalen Netzwerken. Scott identifiziert hier sechs Organisationsprinzipien der transnationalen Kooperation und Governance: 1. die Einrichtung von intergouvernementalen Institutionen zur Schaffung von Foren für staatliche Repräsentant*innen und Vertretungen; 2. inter-institutionelle Foren und NGO-Netzwerke bezüglich spezifischer Themen; 3. globale regionale Entwicklungskonzepte und Handlungspläne, 4. die Existenz und Implementierung von EU-Politiken und Programmen, die zwischenstaatliche Kooperation auf allen Ebenen fördern und Anreize schaffen; 5. lokale Projekte und Initiativen; sowie 6. digitale Vernetzung und Infrastrukturen (vgl. ebd., S. 137). Diese Formen und Foren sowie Bedingungen für grenzüberschreitende Governance erweisen sich als essenziell, um eine effektive und effiziente Form der politischen Steuerung und gleichzeitig territoriale, wirtschaftliche und soziale Kohäsion in der transnationalen Makroregion zu fördern.

Die Studie von Scott veranschaulicht, wie auch auf größeren Skalen, Territorien und höheren administrativen Ebenen, zwischenstaatliche Politik in der Raumentwicklung durch politische Steuerung und Koordination verschiedener Akteur*innen vollzogen wird. Nationale Regierungen sind nicht allein in multilateralen transnationalen Netzwerken (wie dem Ostseeraum-Netzwerk) tätig, sondern delegieren Kompetenzen und Aufgaben auf ihre subnationalen Gebietskörperschaften, die wiederum das Netzwerk auch für NGOs, Akteur*innen aus Wissenschaft und Wirtschaft öffnen, mit dem Ziel, Raumentwicklung in der Region anzustoßen. Die kol- 
laborative politische Steuerung durch gemeinschaftliche Organe wird dann als Governance bezeichnet.

\subsection{Fallbeispiel 4: TransOderana EVTZ}

Eine vierte Fallstudie an der deutsch-polnischen Grenze untersucht grenzüberschreitende Governance im Prozess der Verrechtlichung und Institutionalisierung von grenzübergreifenden Territorialverbünden anhand der von der EU 2006 eingeführten EU-Rechtsform des EVTZ (Europäischer Verbund für territoriale Zusammenarbeit; vgl. Krzymuski et al. 2017).

Entlang der ehemaligen Preußischen Ostbahnstrecke, die im Zweiten Weltkrieg zerstört worden ist und zum Teil reaktiviert wurde, ist eine Modellregion geplant, die nicht nur eine höhere Mobilität und Anbindung garantiert, sondern auch zu einer wirtschaftlichen Entwicklung entlang der Bahnstrecke beitragen soll. Diese Modellregion soll die EU-Rechtsform des EVTZ annehmen und als Mitglieder neben Städten und Gemeinden auch Landkreise sowie auf polnischer Seite eine staatliche Fachhochschule involvieren (vgl. Ulrich 2017, S. 394). Die Mitglieder wären bei erfolgreichem Abschluss eines grenzüberschreitenden EVTZ Akteur"innen in einer intra-institutionellen grenzüberschreitend-supraregionalen Governance-Struktur. Um aber zu einer intra-institutionellen Governance in einer supraregionalen Struktur zu kommen, bedarf es einer inter-institutionellen grenzüberschreitenden Aushandlung von zahlreichen verschiedenen staatlichen und nichtstaatlichen Akteur*innen. Die Idee der Gründung eines EVTZ ist bottom-up durch eine zivilgesellschaftliche Organisation Interessengemeinschaft Ostbahn e.V. (IGOB) entstanden, die über regionale Projektmanager, Gemeinden, Landkreise und mit Unterstützung von Akteur*innen aus Brüssel und wissenschaftlicher Expertise von regionalen wissenschaftlichen Einrichtungen vorbereitet wurde. Die finale Gründung und Vertiefung der Kooperationsstrukturen wurden aber bisher nicht von den staatlichen Bewilligungsbehörden abgeschlossen. Dies zeigt zum einen, dass nationale Grenzen des Rechts und der Verwaltung durchaus ein verhinderndes Element sein können - in diesem Fall sind es rechtliche, politische und administrative Grenzen (vgl. ebd., S. 402ff.).

Die letzte Beispielstudie zeigt einen misslungenen Fall der Institutionalisierung von supraregionaler Governance in EU-Grenzregionen. Obwohl mit der Schaffung dieser Institution Governance-Prozesse im intra-institutionellen grenzüberschreitend-supraregionalen Kontext (innerhalb einer grenzüberschreitenden Organisation) anvisiert wurden, ist aufgrund von nationalen Grenzziehungsprozessen eher inter-institutionelle grenzüberschreitende Governance vonnöten gewesen.

Die oben genannten empirischen Anwendungsfälle stellen Versuche dar, den Begriff der CrossBorder Governance anhand verschiedener empirischer Beispiele zu erläutern. Abschließend soll Cross-Border Governance kritisch evaluiert werden.

\section{Fazit: Kritik und weiterführende Entwicklungsmöglichkeiten}

Das Konzept der Cross-Border Governance hat sich zu Beginn des Jahrtausends im Zuge des europäischen Integrationsprozesses als eines der gewichtigen Konzepte in den politgeografischen Grenzraumstudien etabliert. In vielen Studien wurde Cross-Border Governance angewandt - entweder als analytisches Modell oder Konzept, um die politische Steuerung über 
nationale Grenzen hinweg zu erfassen. Der Hauptkritikpunkt an dem Begriff richtet sich dabei nicht auf den Grenzbezug, sondern auf den viel zu unklaren und schablonenhaften Begriff Governance. Im Folgenden werden beide Termini kritisch reflektiert.

\subsection{Zum Konzept Governance}

Die Grenzen von Governance offenbaren sich bei der definitorischen Spezifizierung, der Form der Anwendbarkeit, der Theoriehaftigkeit und der Zeitgemäßheit des Begriffs. Erstens hat das Konzept Governance keine einheitliche und vorzeigbare, sondern zahlreiche und sehr unterschiedliche Definitionen, die aber meistens auf denselben Prämissen beruhen. Zweitens stellt sich die Frage, wie der Begriff verwendet werden soll. Definiert Governance Normen für gute Politikführung (normativ-ontologischer Begriff) oder dient sie zur empirischen Analyse von Akteur*innen, Netzwerken und Interaktionen (empirisch-analytischer Begriff; vgl. Beck 2017, S. 351f.)? Will man abstrakte Mechanismen aufzeigen und diskutieren oder konkrete Politikregime untersuchen (vgl. Fürst 2007, S. 355)? Soll Governance eine neue Analyseperspektive sein (die bisher in Theorien vernachlässigt worden ist) oder dient Governance als bloße Beschreibung oder empirische Erklärung der Gegenwart europäischen/globalen und grenzüberschreitenden Regierens im postnationalen Raum, die eine neue Form von analytischem Zugang braucht (vgl. ebd., S. 355f.)? Drittens ist der theoretische Gehalt des Begriffs stark infrage zu stellen. Governance wird eher als ein theoretisches Modell oder Theorieansatz verstanden, da sie wenig Erklärungsansätze und generalisierbare Aussagen über die Welt bietet. Daher stellt sich bei Governance stets die Frage: Ist es „[n]ur eine eingängige Metapher oder schon die Vorstufe einer Theorie“ (Knodt/Große Hüttmann 2012, S. 196)?

\subsection{Zum Konzept Cross-Border Governance}

Die oben dargestellten Ausführungen zum Begriff der Cross-Border Governance zeigen auf, dass auch dieser Begriff äußerst unscharf ist und teilweise anders verstanden und angewendet wird. Der Cross-Border Governance-Ansatz fokussiert die netzwerkartige Steuerung nationalstaatlicher bzw. territorialer Grenzen und die mit ihnen zusammenhängenden administrativen, ökonomischen, sozialen und politischen Unterschiede. Egal ob es sich um EU-Binnengrenzen oder EU-Außengrenzen handelt, der Ausgangspunkt ist, dass diese vermeintlich permeabel wahrgenommenen Grenzen durch politische Steuerung in einem Akteur*innennetzwerk in Bezug auf Planung und Umsetzung funktional besser gelenkt und gesteuert werden. Im Falle der EU-Außengrenzen soll eine Durabilität der Grenze durch Ausdifferenzierung von Zuständigkeiten, Akteur*innen, Technologien, Infrastrukturen und weiteren Kontrollinstanzen erreicht werden, während an EU-Binnengrenzen eine weitere Permeabilisierung durch wirtschaftliche, soziale und territoriale Kohäsion und dadurch auch Inklusion zahlreicher Akteur*innen anvisiert wird.

Letztlich stellt sich beim Cross-Border Governance-Ansatz die Frage nach der Aktualität - also wie zeitgemäß ist dieser Erklärungsansatz? Verstehen wir Cross-Border Governance als ein auf neofunktionalistischen und postnationalen Prämissen beruhendes Erklärungsangebot europäisch-regionaler, lokaler oder subnational-grenzüberschreitender Gemeinschaftspolitik, so stellt 
sich unvermeidlich die Frage, inwieweit die ,Schwäche des Nationalstaats‘ noch den tatsächlichen Gegebenheiten entspricht. Mit dem Blick auf die zunehmenden Renationalisierungsprozesse und protektionistischen Diskurse und Praktiken, Handlungen und Rechtsetzungen der Nationalstaaten in Zeiten der Migrations- und der aktuellen Corona-Krise, ist durchaus zu hinterfragen, ob nicht mehr von nation-state failure, sondern von Governance failure (vgl. Jessop 1998, S. 29) gesprochen werden kann. Sind es nicht gerade wieder die Nationalstaaten, die die Agenda setzen und Governance ins Hintertreffen geraten lassen? So wird auch in Grenzregionen im Zuge der Corona-Krise deutlich, dass Nationalstaaten alleinig entscheiden und das Notfallmanagement individuell und unkoordiniert mit subnationalen Akteur*innen und den Nachbarländern vollziehen. Von einer grenzüberschreitenden Governance kann hier nicht die Rede sein. In den meisten Governance-Ansätzen wird häufig von der Ohnmacht der Nationalstaaten ausgegangen. Sind diese protektionistisch-renationalistischen Tendenzen tatsächlich sukzessive eintretend, stellt sich die Frage, ob postnationalistische und neofunktionalistische Prämissen - wie im Governance-Ansatz postuliert - noch zeitgemäß sind. Nach den abrupten Grenzschließungen im März 2020 im Zuge der Corona-Krise zeigte sich jedoch schnell, dass unterstützt durch die EU auch grenzüberschreitende Lösungen gesucht wurden, etwa in der Gesundheitsversorgung oder auf dem Arbeitsmarkt. Die Grenzen wurden dabei für einige Personengruppen sukzessive permeabel und Kooperationen in einigen Politikbereichen durch staatliche und private Akteur*innen vorangetrieben und gelenkt. Des Weiteren haben auch die zivilgesellschaftlichen und bürgerschaftlichen Proteste gegen die Grenzschließung am 24.4.2020 in Frankfurt-Słubice einen Beitrag geleistet, um Kooperation und partielle Mobilität im Grenzraum wieder zu erzwingen und einer einseitigen staatlichen Grenzschließung entgegenzutreten. Solche Fragestellungen können auch in weiterführenden Studien untersucht werden. Welche Rolle spielen grenzüberschreitende regionale oder lokale Regierungsnetzwerke, wer sind ihre Akteur*innen und in welchen Bereichen sind diese Netzwerke integriert? Wie resilient sind diese Netzwerke in Zeiten von Krisen, Renationalisierung und rebordering? Und wie verhalten sich Zentrum und Peripherie zueinander in Zeiten von Krisen?

Auch diese aktuellen Entwicklungen im Zuge der Corona-Krise zeigen, dass Cross-Border Governance in Bezug auf politische grenzüberschreitende Steuerungsprozesse die einzige praktikable Lösung in Planungs- und Politikkontexten auf grenzregionaler Ebene ist, da sie flexibel ist, Lernprozesse ermöglicht und den partizipierenden Akteur*innen auch großen Spielraum liefert. In Bezug auf grenzüberschreitend-planungspolitische Prozesse ist Cross-Border Governance daher umsichtiger als Cross-Border Government.

\section{Weiterführende Literatur}

Benz, Arthur/Dose, Nicolai (Hrsg.) (2010): Governance - Regieren in komplexen Regelsystemen. 2., überarb. Aufl., Wiesbaden: VS Verlag für Sozialwissenschaften.

Gualini, Enrico (2003): Cross-border Governance: Inventing Regions in a Trans-national Multi-level Polity. In: disP - The Planning Review 39, H. 152, S. 43-52.

Kramsch, Olivier/Hooper, Barbara (2004): Cross-Border Governance in the European Union. London: Routledge.

Perkmann, Markus/Sum, Ngai-Ling (Hrsg.) (2002): Globalization, Regionalization and Cross-Border Regions. Basingstoke: Palgrave Macmillan.

Scott, James W. (2002): Transboundary Governance in the Baltic Sea Region: Emerging Patterns, Preliminary Results. In: Regional and Federal Studies 12, H. 4, S. 135-153. 


\section{Literaturverzeichnis}

Banse, Christian (2013): Nationale Grenzerfahrungen und grenzüberschreitende Prozesse. Eine soziologische Untersuchung an ausgewählten Grenzregionen. Frankfurt/M.: Peter Lang.

Beck, Joachim (2017): Der EVTZ und seine Akteure - Territoriale Entwicklungssteuerung im Kontext transnationaler Institutionenbildung. In: Krzymuski, Marcin/Kubicki, Philipp/Ulrich, Peter (Hrsg.): Der Europäische Verbund für Territoriale Zusammenarbeit. Instrument der grenzübergreifenden Zusammenarbeit nationaler öffentlicher Einrichtungen in der Europäischen Union. Baden-Baden: Nomos, S. 343369.

Benz, Arthur/Dose, Nicolai (Hrsg.) (2010): Governance - Regieren in komplexen Regelsystemen. 2., überarb. Aufl., Wiesbaden: VS Verlag für Sozialwissenschaften.

Blatter, Joachim (2006): Governance als transdisziplinäres Brückenkonzept für die Analyse von Formen und Transformationen politischer Steuerung und Integration. In: Bogumil, Jörg/Jann, Werner/Nullmeier, Frank (Hrsg.): Politik und Verwaltung. Wiesbaden: VS Verlag für Sozialwissenschaften, S. 50-76.

Fürst, Dietrich (2007): Regional Governance. In: Benz, Arthur/Lütz, Susanne/Schimank, Uwe/Simonis, Georg (Hrsg.): Handbuch Governance. Theoretische Grundlagen und empirische Anwendungsfelder. Wiesbaden: VS Verlag für Sozialwissenschaften, S. 353-365.

Gualini, Enrico (2003): Cross-border Governance: Inventing Regions in a Trans-national Multi-level Polity. In: disP - The Planning Review 39, H. 152, S. 43-52.

Haas, Ernst B. (1968): The uniting of Europe. Political, social, and economic forces 1950 - 1957. Stanford: Stanford University Press.

Hess, Sabine/Kasparek, Bernd (2010): Grenzregime. Diskurse, Praktiken, Institutionen in Europa. Berlin: Assoziation A.

Hooghe, Liesbeth/Marks, Gary (2003): Unravelling the Central State, but how? Types of Multi-Level Governance. In: American Political Science Review 97, H. 2, S. 233-243.

Jachtenfuchs, Markus/Kohler-Koch, Beate (2010): Governance in der Europäischen Union. In Benz, Arthur/Dose, Nicolai (Hrsg.): Governance - Regieren in komplexen Regelsystemen. 2., überarb. Aufl., Wiesbaden: VS Verlag für Sozialwissenschaften, S. 69-93.

Jańczak, Jarosław (2011): Cross-border Governance in Central European Border Twin Towns. Between De-bordering and Re-bordering. In: Ders. (Hrsg.): De-bordering, Re-bordering and Symbols on the European Boundaries. Berlin: Logos Verlag, S. 37-52.

Jessop, Bob (1998): The rise of Governance and the risks of failure: the case of economic development. In: International Social Science Journal 50/155, S. 29-45.

Jessop, Bob (2015): Territory, Politics, Governance and Multispatial MetaGovernance. In: Territory, Politics, Governance 4, H. 1, S. 8-32.

Keating, Michael (2002): Territorial Politics and the New Regionalism. In: Heywood, Paul/Jones, Erik/ Rhodes, Martin (Hrsg.): Development in West European Politics. 2., überarb. Aufl., Basingstoke: Palgrave Macmillan, S. 201-220.

Knodt, Michèle/Große Hüttmann, Martin (2005): Der Multi-Level Governance Ansatz. In: Bieling, HansJürgen/Lerch, Marika (Hrsg.): Theorien der europäischen Integration. Wiesbaden: VS Verlag für Sozialwissenschaften, S. 223-249.

Kohler-Koch, Beate/Quittkat, Christine (2013): De-mystification of participatory democracy. EUGovernance and civil society. Oxford: Oxford University Press.

Kramsch, Olivier/Hooper, Barbara (Hrsg.) (2004): Cross-Border Governance in the European Union. London: Routledge.

Krzymuski, Marcin/Kubicki, Philipp/Ulrich, Peter (Hrsg.) (2017): Der Europäische Verbund für territoriale Zusammenarbeit. Instrument der grenzübergreifenden Zusammenarbeit nationaler öffentlicher Einrichtungen in der Europäischen Union. Baden-Baden: Nomos.

Leresche, Jean-Philippe/Saez, Guy (2002): Political Frontier Regimes: Towards Cross-Border Governance? In: Perkmann, Markus/Saez, Ngai-Ling (Hrsg.): Globalization, Regionalization and Cross-Border Regions. Basingstoke: Palgrave Macmillan, S. 77-99.

Lindberg, Leon N. (1963): The political dynamics of European economic integration. Stanford: Stanford University Press.

Maier, Johannes (2009): Rechtliche Hindernisse für die Implementierung des EVTZ-Instruments in die föderale Verfassungsstruktur Österreichs. In: Jahrbuch des Förderalismus 2009. Baden-Baden: Nomos, S. 455-468.

Moravcsik, Andrew (1998): The choice for Europe. Social purpose and state power from Messina to Maastricht. 2., überarb. Aufl., Ithaca: Cornell University Press.

Perkmann, Markus/Sum, Ngai-Ling (Hrsg.) (2002): Globalization, Regionalization and Cross-Border Regions. Basingstoke: Palgrave Macmillan. 
Perkmann, Markus (2007): Policy entrepreneurship and multi-level Governance: a comparative study of European cross-border regions. In: Environment and Planning C 25, H. 6, S. 861-879.

Pikner, Tarmo (2008): Reorganizing Cross-Border Governance Capacity. The Case of the Helsinki-Tallinn Euregio. In: European Urban and Regional Studies 15, H. 3, S. 211-227.

Rosenau, James/Czempiel, Ernst Otto (1992): Governance without Government: Order and Change in World Politics. Cambridge: Cambridge University Press.

Rhodes, Roderick A. W. (1996): The New Governance: Governing without Government. In: Political Studies XLIV, S. 652-667.

Sassen, Saskia (2013) Deciphering the Global: Its Scales, Spaces and Subjects. London: Taylor \& Francis.

Scott, James W. (1999): European and North American Contexts for Cross-border Regionalism. In: Regional Studies 33, H. 7, S. 605-617.

Scott, James W. (2000a): Euroregions, Governance and Transborder Co-operation Within the EU. In: European Research in Regional Science 10 (Border, Regions and People), S. 104-115.

Scott, James W. (2000b): Transboundary Co-operation on Germany's Borders: Strategic Regionalism through Multilevel Governance. In: Journal of Borderlands Studies 15, H. 1, S. 143-167.

Scott, James W. (2002): Transboundary Governance in the Baltic Sea Region: Emerging Patterns, Preliminary Results. In: Regional and Federal Studies 12, H. 4, S. 135-153.

Scott, James W. (2004): The Northern Dimension: „Multiscalar“ Regionalism in an Enlarging European Union? In: Kramsch, Olivier/Hooper, Barbara (Hrsg.): Cross-Border Governance in the European Union. London: Routledge, S. 135-156.

Svensson, Sara (2013): Forget the Policy Gap: Why local governments really decide to part in cross-border cooperation initiatives. In: Eurasian Geography and Economics 54, H. 4, S. 409-422.

Ulrich, Peter (2017): Grenzüberschreitende funktionale Kooperation im deutsch-polnischen Grenzraum am Beispiel des TransOderana EVTZ - Akteure, Strategien, Institutionen. In: Krzymuski, Marcin/Kubicki, Philipp/Ders. (Hrsg.): Der Europäische Verbund für Territoriale Zusammenarbeit. Instrument der grenzübergreifenden Zusammenarbeit nationaler öffentlicher Einrichtungen in der Europäischen Union. Baden-Baden: Nomos, S. 369-416.

Ulrich, Peter/Krzymuski, Marcin (2018): Actor's Participation in Cross-Border Governance Structures at the German-Polish Border. Case Studies from the Viadrina Region. In: Wassenberg, Birte (Hrsg.): Castle Talks on Cross-Border Cooperation. Fear of Integration? The Pertinence of the Border. Stuttgart: Franz Steiner Verlag, S. 153-183.

Veggeland, Noralv (2004): Post-national Governance and transboundary regionalization: spatial partnership formations as democratic exit, loyalty and voice options? In: Kramsch, Olivier/Barbara Hooper (Hrsg.): Cross-Border Governance in the European Union. London: Routledge, S. 157-170.

Williamson, Oliver E. (1996): The Mechanisms of Governance. Oxford: Oxford University Press.

Wolf, Dieter (2005): Neo-Funktionalismus. In: Bieling, Hans-Jürgen/Lerch, Marika (Hrsg.): Theorien der europäischen Integration. Wiesbaden: VS Verlag für Sozialwissenschaften, S. 65-91.

Zürn, Michael (2016): Democratic Governance beyond the Nation-State. In: European Journal of International Relations 6, H. 2, S. 183-221. 